\title{
Polaritonic network as a paradigm for dynamics of coupled oscillators
}

\author{
Kirill P. Kalinin ${ }^{1}$ and Natalia G. Berloff ${ }^{2,1, *}$ \\ ${ }^{1}$ Department of Applied Mathematics and Theoretical Physics, University of Cambridge, Cambridge CB3 OWA, United Kingdom \\ ${ }^{2}$ Skolkovo Institute of Science and Technology, Bolshoy Boulevard 30, building 1, Moscow 121205, Russian Federation
}

(Received 7 February 2019; revised manuscript received 20 November 2019; published 26 December 2019)

\begin{abstract}
Photonic and polaritonic lattices were recently theoretically proposed and experimentally realized as manybody simulators due to the rich behaviors exhibited by such systems at the macroscale. We show that the networks of polariton condensates encapsulate a large variety of behaviors of systems of coupled oscillators. By eliminating spatial degrees of freedom in a nonresonantly pumped polariton network, we establish that depending on the values of experimentally tunable parameters the networks of polariton condensates may represent Kuramoto, Sakaguchi-Kuramoto, Stuart-Landau, or Lang-Kobayashi oscillators and beyond. The networks of polariton condensates are therefore capable of implementing various regimes acting as analog spin Hamiltonian minimizers, producing complete and cluster synchronization, exotic spin glasses, and large-scale secondary synchronization of oscillations. We suggest that the recently implemented control of the system parameters for individual sites in polariton lattices allows addressing the interaction of sublattices that belong to different oscillatory classes.
\end{abstract}

DOI: 10.1103/PhysRevB.100.245306

\section{INTRODUCTION}

For a long time, two pervasive topics of modern sciencethe dynamics of coupled oscillators and simulations of manybody solid-state systems- - have barely crossed each other's paths. Complex dynamic behavior of networks of coupled oscillators arises in various scientific disciplines ranging from biology, physics, and chemistry to social and neural networks as well as in established and emerging technological applications. Such networks served as paradigmatic models for understanding the mechanism of various collective phenomena. For instance, Kuramoto oscillators [1,2] have been successfully used to represent, study, or even predict a wide variety of pattern formation in spatiotemporal systems, such as biochemical systems, neural networks, convecting fluids, and laser arrays. The reason for such power of networks of coupled oscillators in describing vastly different systems lies in the underlying symmetries of the system: these are described by similar universal order parameter equations that share similar characteristics [3]. Such symmetries make it possible to divide systems into various universality classes that differ only by the nature of the dynamics [4] and allow one not only to draw similarities between very different physical systems but also to predict the behavior of the new systems that fall into previously known universality class [5].

Traditionally, at the other end of the spectrum of nonlinear dynamical studies lie the complex many-body solid-state systems that are often considered powerful platforms for simulating various elaborate Hamiltonians. A number of systems were realized implementing lattices of various physical origins: neutral atoms, ions, electrons in semiconductors, polar

\footnotetext{
*Author to whom correspondence should be addressed: N.G.Berloff@damtp.cam.ac.uk
}

molecules, superconducting circuits, nuclear spins, etc. [6]. These are typically equilibrium systems that realize ground or excited states of their structure Hamiltonians. Recently, photonic and polaritonic lattices have emerged as promising platforms for many-body quantum and classical simulations $[7,8]$. These systems are typically of a gain-dissipative nature, are capable of symmetry breaking and spontaneous pattern forming, and have constant nonzero particle fluxes even in the steady state. Furthermore, we argue in this paper that if the lattice elements have a photonic component and the gain-dissipative nature, then the complex amplitudes characterizing such elements evolve, interact, and synchronize in close resemblance to the coupled oscillators that are governed by the universal order parameter equations. Therefore, on the one hand, many classical phenomena found in such lattices can be explained or predicted by the behavior of the corresponding system of coupled oscillator networks from the same universality class; on the other hand, strong nonlinearities, spin polarization, sensitivity to magnetic fields, and individual site control greatly enrich possible states and dynamical regimes that can be generated in such lattices. In this paper, we propose and theoretically justify the use of networks of exciton-polaritons (polaritonic networks) as a flexible universal platform to realize a vast array of known and extensively studied systems of coupled oscillators to probe exotic dynamical regimes and to create states of matter that may result from hybridization of several different networks on one platform. In particular, we find the states that suggest possible practical implementations towards optical transistors and clarify the requirements for such a network to minimize classical spin Hamiltonians.

In the last decade, it has emerged that strong light-matter interactions in semiconductor microcavities offer a versatile platform to realize nontrivial states, dynamics, and localized structures. They consist of exciton-polaritons (polaritons) that 
are bosonic quasiparticles with a tiny effective mass which is typically $10^{-4}-10^{-5}$ of the bare exciton mass. Their energymomentum dispersion curves can be controlled by appropriate detuning, and their properties and dynamics can be readily accessed by angular-resolved photo- or electroluminescence spectroscopy. A wealth of experimental results has been demonstrated with these systems, including Bose-Einstein condensates (BECs) [9], polariton lasers [10], polariton parametric amplifiers [11], and cavity quantum electrodynamics [12]. The polariton BEC or lasing has been demonstrated in various materials such as CdTe [9], GaAs [13,14], GaN [15], and organic polymers [16] and using optical pumping or electrically pumped exciton-polariton emitters [17].

We are interested in networks of $N$ polariton condensates created at lattice sites: the vertices of a two-dimensional graph at positions $\mathbf{r}_{i}, i=1, \ldots, N$. Many techniques are available to engineer a variety of the potential landscapes of polaritons $[18,19]$. Polaritons can be confined by strain-induced traps [14], surface acoustic waves [20], direct fabrication with the gold deposition technique [21], using hybrid air gap microcavities [22], or coupled mesas etched during the growth of the microcavity [23], by micropillars [24], and in various geometries: square [21], triangular [25], hexagonal [26], a fully etched honeycomb [27], kagome [28], and even quasiperiodic potentials [29]. However, the potential traps in a gain-dissipative system lead to complicated dynamics as the flow dynamics present in gain-dissipative systems even in the steady state is highly nontrivial in this geometry. To avoid such complications, polariton lattices can be optically engineered by exploiting the interactions between polaritons and reservoir excitons that can be injected in specific areas of the sample. Excitons barely move from the point where they are excited as they are orders of magnitude heavier than polaritons. Experimentally, the lattice is achieved by using a spatial light modulator that creates polariton condensates at the vertices of any prescribed graph [30-34]. This technique also allows controlling the pumping intensities $p_{i}$ of individual sites indexed by $i$ depending on the density of the polariton condensate at this site, if needed. In what follows we derive and discuss the network behavior bearing in mind this technique of the lattice formation; however, other ways to create polariton lattices affect the parameters but not the universality of the derived coupled oscillators equations.

\section{MEAN-FIELD MODELS OF POLARITONIC NETWORKS AND COUPLED OSCILLATORS}

The mean-field behavior of polariton condensates is described by the generalized complex Ginzburg-Landau equation (often also referred to as a driven-dissipative Gross-Pitaevskii equation) coupled to the reservoir dynamics [35-38]. Although the process of Bose-Einstein condensation includes quantum effects, when a condensate is formed, it is accurately described by the mean-field equations, as was shown in numerous experimental works [33,34,39-45]. The equation on the wave function $\psi(\mathbf{r}, t)$ of the condensed system is coupled to the rate equation on the density of the hot reservoir $n_{R}(\mathbf{r}, t)$, so that $i \psi_{t}=-\frac{1}{2 m}\left(1-i \hat{\eta} n_{R}\right) \nabla^{2} \psi+U_{0}|\psi|^{2} \psi+g_{R} n_{R} \psi+$ $\frac{i}{2}\left[R_{R} n_{R}-\gamma_{C}\right] \psi$, and $n_{R t}=-\left(\gamma_{R}+R_{R}|\psi|^{2}\right) n_{R}+P(\mathbf{r}, t)$, where we set $\hbar=1, U_{0}$ and $g_{R}$ are the polariton-polariton and polariton-exciton interaction strengths, respectively, $\hat{\eta}$ is the energy relaxation $[5,46]$, and $R_{R}$ is the rate of scattering from the hot reservoir into the condensates. The condensate $\left(\gamma_{C}\right)$ and the reservoir $\left(\gamma_{R}\right)$ relaxation rates describe photon losses in the cavity and hot exciton losses other than scattering into condensates. The incoherent pump source is described by the pumping intensity $P(\mathbf{r}, t)$. We nondimensionalize these equations by $\psi \rightarrow \sqrt{\gamma_{C} / 2 U_{0}} \psi$, $t \rightarrow 2 t / \gamma_{C}, \mathbf{r} \rightarrow \sqrt{1 / m \gamma_{C}} \mathbf{r}, n_{R} \rightarrow \gamma_{C} n_{R} / R_{R}, P \rightarrow P \gamma_{C}^{2} / 2 R_{R}$ and introduce the dimensionless parameters $g=2 g_{R} / R_{R}$, $b_{0}=2 \gamma_{R} / \gamma_{C}, b_{1}=R_{R} / U_{0}, \eta=\hat{\eta} \gamma_{C} / R_{R}$. The resulting model yields

$$
\begin{aligned}
& i \frac{\partial \psi}{\partial t}=-\left(1-i \eta n_{R}\right) \nabla^{2} \psi+|\psi|^{2} \psi+g n_{R} \psi \\
&+i\left(n_{R}-1\right) \psi \\
& \frac{\partial n_{R}}{\partial t}=-\left(b_{0}+b_{1}|\psi|^{2}\right) n_{R}+P(\mathbf{r}, t) .
\end{aligned}
$$

A unique property of the exciton-polariton system is the flexibility with which the parameters $g, b_{0}, b_{1}, \eta$ can be controlled and changed. Exciton-polaritons offer a testbed that bridges the physics of lasers and other nonequilibrium systems with equilibrium condensates as well as allows one to explore novel physical regimes. The lifetime of polaritons $\gamma_{C}$ is controlled by the accuracy of the cavity distributed Bragg reflectors and spans two orders of magnitude $[9,47]$. The detuning between the cavity photon energy and the exciton resonance determines the proportion of photon and exciton in the polariton and therefore the strength of the polariton-polariton and polariton-exciton interactions and effective mass $[37,38]$. The repulsive interactions between excitons and polaritons $g_{R}$ can be further tuned by the pumping geometry, for instance, considering trapped condensates separated from the pumps [39].

The building block of our network is a single stationary condensate described by a wave function $\psi=\phi(r)$, created by a spatially localized radially symmetric incoherent pumping source $P=p(r)$. For instance, a Gaussian pump $p(r)=A \exp \left[-w r^{2}\right]$, where $w$ determines the inverse width, has been widely used in experiments $[30,34]$. In what follows we assume that the pumping intensity $A$ is chosen so that $\phi$ is normalized, so that $\int_{Q}|\phi|^{2} d \mathbf{r}=1$, where $Q$ is the entire plane of the cavity. We define the corresponding stationary reservoir profile as the steady state of Eq. (2), so that $n_{R}(r)=n(r)=p /\left(b_{0}+b_{1}|\phi|^{2}\right)$. The networks of $N$ polariton condensates are created at lattice sites $i$ using a time- and space-varying pumping profile $P(\mathbf{r}, t)=$ $\sum_{i=1}^{N} f_{i}(t) p\left(\left|\mathbf{r}-\mathbf{r}_{i}\right|\right)$. The total wave function $\psi$ and the reservoir density $n_{R}$ can be approximated by $\psi(\mathbf{r}, t) \approx$ $\sum_{i=1}^{N} a_{i}(t) \phi\left(\left|\mathbf{r}-\mathbf{r}_{i}\right|\right)$ and $n_{R}(\mathbf{r}, t) \approx \sum_{i=1}^{N} k_{i}(t) n\left(\left|\mathbf{r}-\mathbf{r}_{i}\right|\right)$, respectively. Such an approximation is valid if the distance between the lattice sites exceeds the width of the condensate and reservoir [48]. This approach is reminiscent of the tight-binding approximation that was applied before to the equilibrium Gross-Pitaevskii equation [49-51]. The main difference between the equations arises from the nature of the driven-dissipative system considered here and from the coupling mechanism: the nearest-neighbor interactions are 
created by the kinetic term in the optical lattices of equilibrium condensates, while in our case the couplings are dominated by the excitonic reservoir while polariton condensates are spatially separated. In the derivations below, we use the shorthand notation $p_{i} \equiv p\left(\left|\mathbf{r}-\mathbf{r}_{i}\right|\right)$, with similar notation for $\phi$ and $n$. We eliminate the spatial degrees of freedom by multiplying Eq. (1) by $\phi_{i}^{*}$, multiplying Eq. (2) by $\left|\phi_{i}\right|^{2}$, and integrating both equations over the plane of cavity $Q$. Previously, we used this approach on a single Ginzburg-Landau equation that is relevant to the description of polariton condensates under several stringent assumptions: negligible blueshift due to interactions of polaritons with the reservoir, short lifetime sample, fast reservoir relaxation, and near-threshold pumping intensity [48]. Now we shall drop such restrictions and show how systems of different universality classes become relevant. We use the smallness of the overlap integrals for the wave functions of the different lattice sites [59] so that $l_{i j} \equiv \int_{Q} n_{i} \phi_{j} \phi_{i}^{*} d \mathbf{r} \gg \int_{Q} \phi_{j} \phi_{i}^{*} d \mathbf{r}, l=l_{i i} \gg$ $\int_{Q} n_{i}\left|\phi_{j}\right|^{2} d \mathbf{r}$, and $H=\int_{Q} n \nabla^{2} \phi \phi^{*} d \mathbf{r} \gg \int_{Q} n_{i} \nabla^{2} \phi_{j} \phi_{i}^{*} d \mathbf{r}$ if $i \neq j$. We also assume that for sufficiently smooth condensate profiles $\int_{Q} n_{i} \nabla^{2} \phi_{j} \phi_{i}^{*} d \mathbf{r} \ll l_{i j}$. The dynamical equations on $\Psi_{i}(t)=a_{i}(t) \exp [-i d t]$ and $R_{i}(t)=l k_{i}$ become

$$
\begin{gathered}
\dot{\Psi}_{i}=-i\left|\Psi_{i}\right|^{2} \Psi_{i}-\Psi_{i}+h R_{i} \Psi_{i}+(1-i g)\left[R_{i} \Psi_{i}\right. \\
\left.+\sum_{j \neq i} J_{i j} \Psi_{j}\right], \\
\quad \dot{R}_{i}=b_{0}\left(\gamma_{i}-R_{i}-\xi R_{i}\left|\Psi_{i}\right|^{2}\right),
\end{gathered}
$$

where we used the notation $d=\int_{Q} \phi^{*} \nabla^{2} \phi d \mathbf{r}, \quad h=$ $\eta H / l, \quad \gamma_{i}=f_{i} \int_{Q} p|\phi|^{2} d \mathbf{r} / b_{0}, \xi=b_{1} \int_{Q} n|\phi|^{4} d \mathbf{r} / l b_{0}, \quad J_{i j}=$ $\left(R_{i} l_{i j}+R_{j} l_{j i}^{*}\right) / l$. The energy relaxation parameter $\eta \ll 1$; therefore, $|H|<l$, so the term $|h| R_{i} \Psi_{i}$ will be neglected in comparison with $R_{i} \Psi_{i}$, whereas the imaginary part of $h$ will be assumed to be absorbed by $g$. As a result, the time-dependent Eqs. (3) and (4) approximate the original Eqs. (1) and (2) with each site in a network of condensates being described as a complex number $\Psi_{i}$, i.e., the condensate's amplitude and phase. For each node we consider nonlinear polariton-polariton interactions $\left(-i\left|\Psi_{i}\right|^{2} \Psi_{i}\right)$, linear losses $\left(-\Psi_{i}\right)$, the exciton conversion rate to polaritons $\left(R_{i} \Psi_{i}\right)$, exciton-polariton interactions $\left(-i g R_{i} \Psi_{i}\right)$, and coupling coefficients between network sites $\left((1-i g) \sum_{j \neq i} J_{i j} \Psi_{j}\right)$. The coupling strength $J_{i j}$ is generally a complex number too, so we write $J_{i j} \equiv \mathcal{J}_{i j} \exp \left[i v_{i j}\right]$ for real $\mathcal{J}_{i j}$ and $v_{i j}$. In deriving Eqs. (3) and (4) we neglected higher-order nonlinearities in $\Psi_{i}$ in view of their smallness close to the condensation threshold. Next, we consider several special cases of Eqs. (3) and (4).

Fast reservoir relaxation limit $b_{0} \gg 1$. In this limit, the reservoir dynamics can be replaced with its steady state, so $R_{i}=\gamma_{i} /\left(1+\xi\left|\Psi_{i}\right|^{2}\right) \approx \gamma_{i}-\xi \gamma_{i}\left|\Psi_{i}\right|^{2}$, reducing the system of Eqs. (3) and (4) to the single equation

$$
\begin{aligned}
\dot{\Psi}_{i}= & i\left(g \xi \gamma_{i}-1\right)\left|\Psi_{i}\right|^{2} \Psi_{i}-\xi \gamma_{i}\left|\Psi_{i}\right|^{2} \Psi_{i}-\Psi_{i} \\
& +(1-i g)\left[\gamma_{i} \Psi_{i}+\sum_{j \neq i} J_{i j} \Psi_{j}\right] .
\end{aligned}
$$

For uniform pumping $\gamma_{i}=\gamma$ this is a celebrated StuartLandau system of coupled oscillators [52]. This model can approximate a wide range of different oscillatory systems as it represents the normal form of an Andronov-Hopf bifurcation. Operating close to an instability threshold, lasers represent an example of the system close to such a bifurcation. We substitute $\Psi_{i}(t)=\sqrt{\rho_{i}(t)} \exp \left[i \theta_{i}(t)\right]$ into Eq. (5) and separate the real and imaginary parts to get

$$
\begin{aligned}
\frac{1}{2} \dot{\rho}_{i}(t)= & \left(\gamma_{i}-1-\xi \gamma_{i} \rho_{i}\right) \rho_{i} \\
& +\sum_{j ; j \neq i} \tilde{J_{i j}} \sqrt{\rho_{i} \rho_{j}} \cos \left(\theta_{i j}-v_{i j}+\alpha\right), \\
\dot{\theta}_{i}(t)= & \left(g \xi \gamma_{i}-1\right) \rho_{i}-g \gamma_{i} \\
& -\sum_{j ; j \neq i} \tilde{\tilde{J}_{i j}} \frac{\sqrt{\rho_{j}}}{\sqrt{\rho_{i}}} \sin \left(\theta_{i j}-v_{i j}+\alpha\right),
\end{aligned}
$$

where $\theta_{i j}=\theta_{i}-\theta_{j}, \quad \tan \alpha=g$, and $\tilde{I_{i j}}=\mathcal{J}_{i j} / \cos \alpha$. Note that for the Gaussian pumping profile and wide reservoir, $\left|v_{i j}\right| \ll\left|\mathcal{J}_{i j}\right|$, so the term $v_{i j}$ can be neglected since $l_{i j} \approx$ $l_{j i}^{*}$ [48]. For other network geometries such an assumption may not be valid, in which case we can absorb $v_{i j}$ into $\alpha_{i j}=\alpha-v_{i j}$.

Experimentally, the feedback can be applied to bring all the sites to the same density $\rho_{i}(t)=\left|\Psi_{i}(t)\right|^{2}=\rho_{\text {th }}$ by combining Eq. (5) with an equation on the pumping adjustments,

$$
\dot{\gamma}_{i}(t)=\epsilon\left[\rho_{\text {th }}-\rho_{i}(t)\right],
$$

where the parameter $\epsilon$ characterizes the rate of such an adjustment or its discrete version applied at discrete times $t_{n}$ (which is more appropriate for the current experimental control techniques), so that $\gamma_{i}\left(t_{n}<t \leqslant t_{n+1}\right)=\gamma_{i}\left(t_{n}\right)$ $+\epsilon\left(t_{n+1}-t_{n}\right)\left[\rho_{\text {th }}-\rho_{i}\left(t_{n}\right)\right]$. Under this control, close to the threshold $\rho_{i} \approx \rho_{\text {th }}$, and Eqs. (6) and (7) reduce to a single equation,

$$
\dot{\theta}_{i}(t)=\left(g \xi \gamma_{i}-1\right) \rho_{\mathrm{th}}-g \gamma_{i}-\sum_{j ; j \neq i} \tilde{J_{i j}} \sin \left(\theta_{i j}+\alpha\right) .
$$

This is the Sakaguchi-Kuramoto model of coupled oscillators [53], with $\alpha$ representing a phase lag. Synchronization and desynchronization in this system have been extensively studied in contexts as vastly different as a network of Wien-bridge oscillators in an experimental regime for which they can be approximated as phase oscillators [54], power grids consisting of many oscillating generators [55], and earthquake sequencing studies [56]. The phase lag appears as a result of synaptic organizations in neuroscience systems, time delays in sensor networks, or transfer conductances in power networks. The Sakaguchi-Kuramoto model is a special case of the Winfree model with $\delta$-function pulse shape $W_{1}(\theta)$ and a sinusoidal response curve $W_{2}(\theta)$, so that $\dot{\theta}_{i}=\omega_{i}+W_{2}\left(\theta_{i}\right) \sum_{i=1}^{N} W_{1}\left(\theta_{j}\right)$. If the coupling is sufficiently weak and the oscillators are nearly identical, the phase can be replaced by its average over an entire period of oscillations, leading to the SakaguchiKuramoto model.

If $g=0(\alpha=0)$, then Eq. (9) reduces to the paradigmatic Kuramoto model $[1,2]$ that was the first tractable mathematical model for describing how coherent behavior emerges in complex systems. This model exhibits a phase transition at a critical coupling, beyond which a collective behavior is achieved. In our case, all natural frequencies are identical (and equal to $\left.\rho_{\mathrm{th}}\right)$ and the equation describes the negative gradient flow $\dot{\theta}=-\partial U(\theta) / \partial \theta$ for the smooth function $U(\theta)=$ 
$-\sum_{i, j}{\tilde{J_{i j}}}_{\cos } \theta_{i j}$. Therefore, by the LaSalle invariance principle (e.g., in [57]) every trajectory converges to a minimum of the XY Hamiltonian $H_{X Y}=-\sum_{i=1}^{N} \sum_{j=1}^{N} \tilde{J_{i j}} \cos \left(\theta_{i}-\theta_{j}\right)$. In the case of $\mathbb{S}^{1}$-synchronizing graphs all critical points are hyperbolic, so the synchronized state is the global minimum of $U(\theta)$, and all other critical points are local maxima or saddle points [58]. For arbitrary graphs the global minimum can be achieved by implementing the lowest pumping intensity that leads to the threshold $\rho_{\text {th }}$ [48]. Polariton graphs as global minimizers of the XY Hamiltonian were theoretically justified and experimentally realized in our previous work [30,59]. Using a resonant pumping in addition to the nonresonant one [adding the terms proportional to $\Psi_{i}^{*}$ to the right-hand side of Eq. (1)] allows one to minimize the Ising or Potts Hamiltonians [60]. Several other driving-dissipative platforms exploited this idea for minimization of spin Hamiltonians: injection-locked laser systems [61], networks of optical parametric oscillators [62-65], coupled lasers [66], and photon condensates [67]. As the analogy with the coupled oscillators we presented above suggests, the minimization of a spin Hamiltonian is realized for the type of coupling that allows for the flow to be represented by the negative gradient flow with real function $U$. This condition is satisfied only if the coupling matrix $J_{i j}$ in Eq. (5) is self-adjoint $\mathcal{J}_{i j}=\mathcal{J}_{j i}$ and $v_{i j}=-v_{j i}$. For instance, if the couplings in Eq. (5) are of a pure Josephson type (e.g., $\dot{\Psi}_{i}=\cdots+i \sum_{j} K_{i j} \Psi_{j}$ with real couplings $K_{i j}$ ) or have a non-negligible $g$, such a network will not necessarily minimize spin or any other Hamiltonian.

In addition, the parameter $g$ has a destabilizing effect on the fixed points of Eq. (9). Different $\gamma_{i}$ that have to be maintained to allow all densities to reach the same value $\rho_{\text {th }}$ provide each lattice element with its own "natural frequency," $\omega_{i}=$ $\left(g \xi \gamma_{i}-1\right) \rho_{\mathrm{th}}-g \gamma_{i}$, and therefore favor desynchronization. In the network described by Eq. (9) synchronization occurs when the coupling dominates the dissimilarity introduced by natural frequencies and the phase lag. The smaller $g$ is, the more likely it is the global synchronization will be achieved. Concise results for complex networks are known for specific topologies such as complete graphs, highly symmetric ring or linear graphs, acyclic graphs, and complete bipartite graphs with uniform weights. Also, the Sakaguchi phase lag parameter $\alpha$ contributes to desynchronization as it provides attraction and repulsion between the oscillator phases similar to the coupling time delay. The dependence of synchronization and desynchronization in polariton condensates on $g$ was noted experimentally, but the reasons have not previously been identified [31-33,68]. Such behavior, however, is easily explained from the point of view of the dynamics of coupled oscillators.

Slow reservoir relaxation limit. Equation (5) describes the direct coupling scheme: pumping at the mean field, calculated algebraically from the states of all oscillators, enters the coupling. The coupling scheme of Eqs. (3) and (4) is more complex: the mean field acts on the reservoir densities that obey its own nonlinear differential equations, and the acting force is a function of the reservoir state. This is similar to the famous example of synchrony on London's Millennium Bridge, where equations for the swinging mode of the bridge are coupled to the equations on individual pedestrians [69], or to electronic or electrochemical oscillators that are coupled

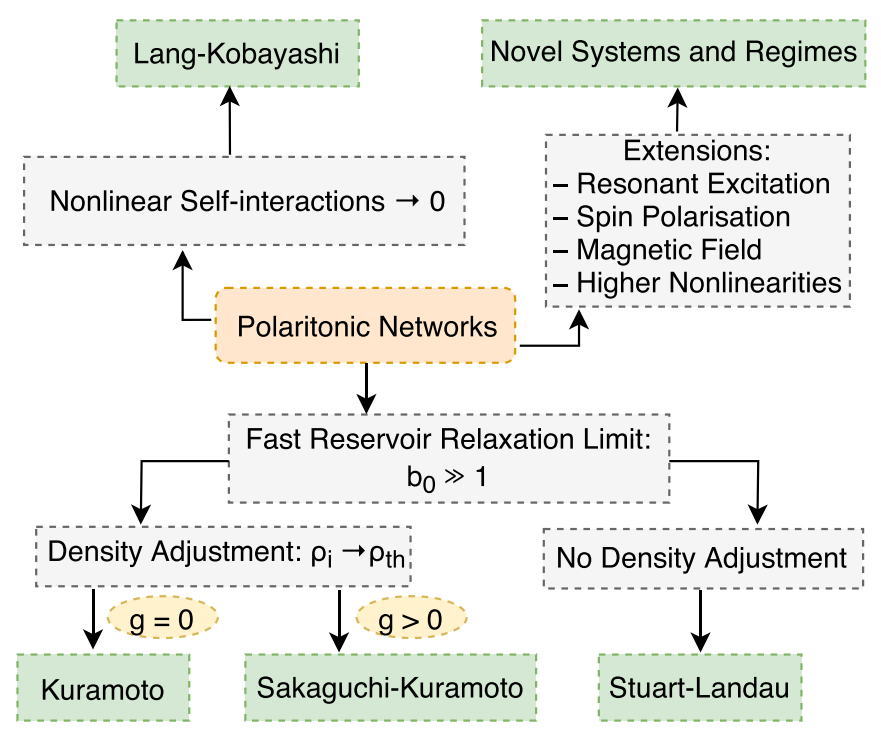

FIG. 1. The polaritonic networks described by Eqs. (3) and (4) can lead to the Lang-Kobayashi model in the absence of the nonlinear self-interaction term or to Kuramoto/Sakaguchi-Kuramoto/StuartLandau models of coupled oscillators in the limit of fast reservoir relaxation. The new regimes are expected to appear due to strong polariton-polariton interactions or once the experimental controls such as resonant excitation pump, spin polarization, and magnetic field or a combination of different sublattices are considered in polaritonic networks.

through the common macroscopic current or voltage, which obeys macroscopic equations describing the coupling circuit [70]. By tuning the photonic component of polaritons one can change the polariton-polariton interactions up to four orders of magnitude [68], which allows one to neglect the term $\left|\Psi_{i}\right|^{2} \Psi_{i}$, so that Eqs. (3) and (4) become similar to the Lang-Kobayashi equations (with $\Psi_{i}$ replaced by the electric field and $R_{i}$ replaced by the population inversion of the $i$ th laser) obtained using Lamb's semiclassical laser theory and are capable of describing the dynamical behavior of coupled lasers [71,72]. We summarize all the regimes and models described above schematically in Fig. 1.

The flexibility to tune the system parameters, the shape and geometry of the polariton lattice [30], the existence and tunability of the nonlocal couplings beyond the next-neighbor interactions [73], and strong self-interactions of polariton condensates allow one to not only recreate the intriguing patterns, states, and structures that have fascinated the nonlinear dynamics community in the last couple of decades but also to enter novel regimes.

\section{REALIZATION OF HYBRID POLARITONIC NETWORKS}

To illustrate a possibility for the exciton-polariton system to enter hybrid regimes, we consider two polaritonic networks configurations in which the dynamic behavior is the result of the interplay between two or more subsystems that belong to different types of coupled oscillators.

Triangular lattice in the fast reservoir relaxation regime with low g, short lifetime, excitonic polariton condensate, and constant pumping. Any finite two-dimensional polariton 
lattice with the same pumping intensity across all the sites gives rise to an example of the interplay between Kuramoto (for $g=0$ ) or Sakaguchi-Kuramoto (for $g \neq 0$ ) oscillators away from the network boundaries and the Stuart-Landau oscillators at the sites close to the boundary. When all the lattice sites are equally pumped, the internal sites have the same number of neighbors and therefore receive equal particle fluxes from them. On the boundary, however, the number of neighbors is diminished, so fewer fluxes bring fewer particles, decreasing the density of the oscillators. The difference in the "natural frequency" [given by the first term on the right-hand side of Eq. (7)] between the boundary and bulk condensates tends to desynchronize the lattice, but for lower pumping intensities this effect is overwhelmed by the synchronization effect of the couplings, so the lattice is frequency synchronized [see Fig. 2(b)]. As the pumping intensity is increased further above the threshold, the frequencies may still be synchronized, but the phases corresponding to a spin glass emerge as shown in Figs. 2(c) and 2(d). The appearance of the spin glass is due to nonlocal coupling and an increasing role of Dzyaloshinskii-Moriya interactions (DMIs) since the density imbalance between the sites makes the interaction directional and therefore of the DMI type [74,75]. For even higher pumping intensities the desynchronization takes place [Figs. 2(e)-2(g)], resulting in a peculiar quasi-one-dimensional form of largescale collective density oscillations in the direction of the larger axis of symmetry of the lattice. The collective density oscillates between the left and right parts of the lattice with the appearance (and disappearance) of the $\pi$-phase difference between two clusters, indicating temporal formation of the domain wall. Figure 2 illustrates these regimes using the full spatially resolved simulations of the polaritonic networks [Eqs. (1) and (2)] in the fast reservoir relaxation limit. These states are robust, sustain external noise, exist for a wide range of parameters, and are reached from random initial conditions. The collective density oscillations also appear when larger lattices are considered. The dimensionless parameters listed in the caption of Fig. 2 correspond to a polariton lifetime of $2 \mathrm{ps}$, exciton reservoir lifetime of $4 \mathrm{ps}, U_{0}=7.9 \mu \mathrm{eV} \mu \mathrm{m}^{2}$ and $g_{R}=0.12 \mu \mathrm{eV} \mu \mathrm{m}^{2}$ (the excitonic component of the polaritons dominates over their photonic component), polariton mass $m=0.4 \mathrm{meV} \mathrm{ss}^{2} / \mu \mathrm{m}^{2}$, and $R_{R}=20 g_{R}$. These parameters fall into experimentally measured and numerically used ranges [42,68,76,77].

We note that the system behavior as the pumping intensity increases is reminiscent of the Mott metal-insulator transition. The frequency of the collective oscillations quenches towards the transition point [see the inset in Fig. 2(a)], after which the oscillation frequency increases as a linear function, repeating the linear trend of the original Mott transition. We can induce reversible changes between these two states of the system by increasing or decreasing pumping intensity. Such oscillations suggest an interesting application towards implementation of polariton transistors [78] that operate in the gigahertz range.

Triangular lattice in both fast and slow reservoir relaxation regimes with low g, short lifetime, photonic polariton condensate, and constant pumping. Our second example concerns cluster synchronization, a particular synchronization phenomenon that requires synchronization to occur in each group,
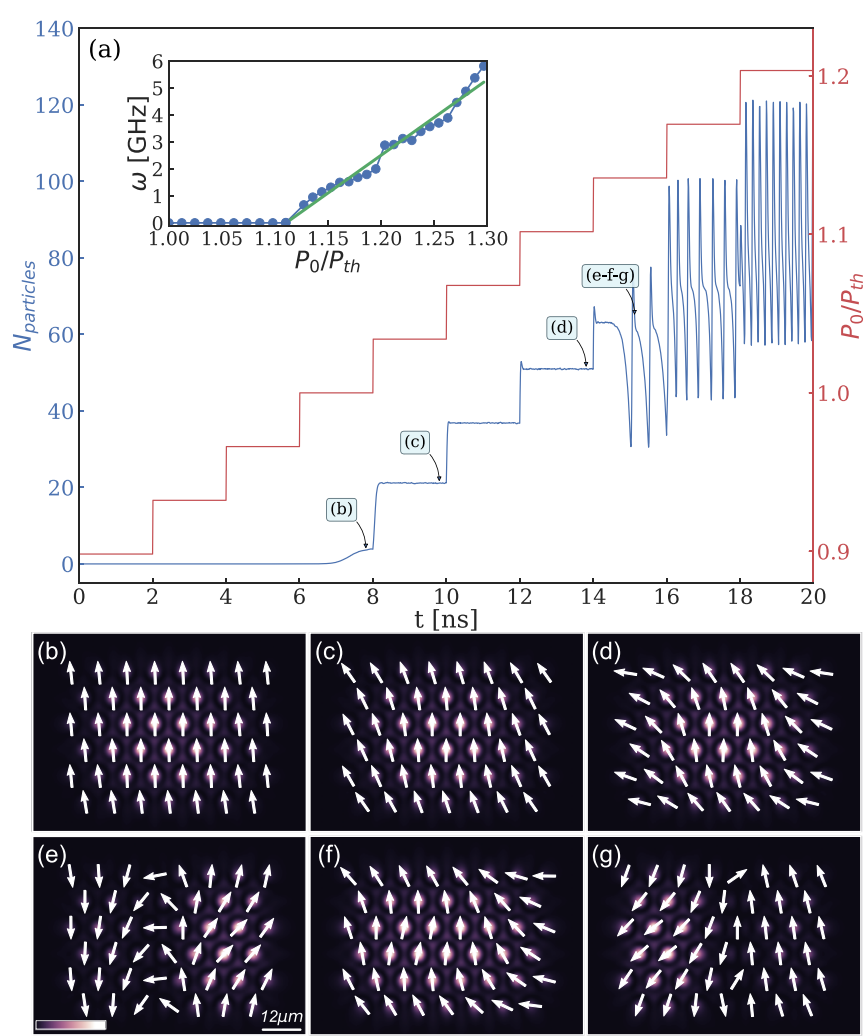

FIG. 2. The results of numerical integration of Eqs. (1) and (2) in the fast reservoir relaxation limit for 45 polariton condensates arranged in the regular triangular lattice with a lattice constant $d=9 \mu \mathrm{m}$. (a) The total number of particles $N_{\text {particles }}$ as a function of time $t$ (blue line). The time-dependent pumping intensity increases above the threshold $P_{0} / P_{\text {th }}$, as shown in red, where $P_{\text {th }}=3.9$. The density distribution and phases of individual condensates are shown in (b)-(g). (b) The density and phases of the stationary lowest-energy state at $P_{\text {th }}$ with ferromagnetic couplings. (c) and (d) The spin configuration changes for the higher pumping intensities as the DMIs become noticeable. (e-g) Time snapshots over the period of density oscillations at $P_{0}=1.14 P_{\text {th }}$ demonstrate the collective density oscillations with a temporary formation of the domain wall. The inset in (a) shows the oscillation frequency of the number of particles $N_{\text {particles }}$ as a function of $P_{0} / P_{\text {th }}$. The transition point between the stationary state and the collective periodic density oscillations is at $P_{0}=1.11 P_{\mathrm{th}}$. The frequency of oscillations increases linearly for $P_{0}>1.11 P_{\text {th }}$. For $P_{0}>1.3 P_{\text {th }}$ the system exhibits chaotic density oscillations. Random initial conditions and white noise were used in numerical simulations with $g=0.1, b_{0}=1, b_{1}=0.3, \eta=0.12$, $w=1.33, P(\mathbf{r})=\sum_{i=1}^{N} P_{0} \exp \left[-w\left(\mathbf{r}-\mathbf{r}_{i}\right)^{2}\right]$.

but there is no synchronization among the different groups [79]. The importance of cluster synchronization has been found in various applications, including biological sciences and communication engineering, and various control schemes were designed to drive the network to cluster synchronization [80]. In polaritonic networks, cluster synchronization presents itself even in the case of equally pumped lattices. To illustrate cluster formation, we consider regular triangular lattices and numerically integrate Eqs. (1) and (2) in two opposite limits: fast [Fig. 3(a)] and slow [Fig. 3(c)] reservoir relaxation times. During a fraction of a nanosecond the system is frequency locked into several clusters; some of them are shown in 


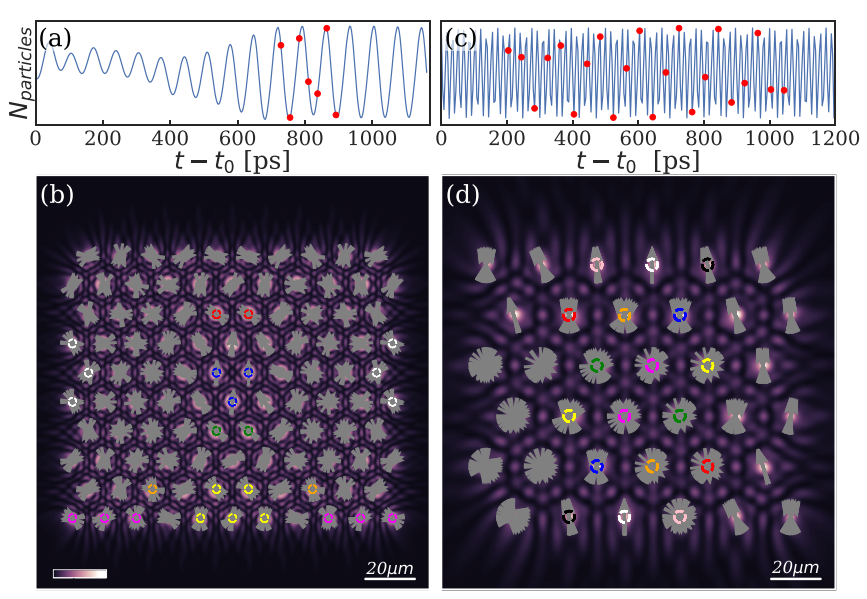

FIG. 3. (a) and (c) The numbers of particles $N_{\text {particles }}$ as a function of time $t-t_{0}$ calculated by numerical integration of Eqs. (1) and (2) in (a) fast and (c) slow reservoir relaxation limits for (a) 105 and (c) 36 polariton condensates arranged in the regular triangular lattice with a lattice constant of (a) $d=13 \mu \mathrm{m}$ and (c) $d=$ $22.5 \mu \mathrm{m}$. (b) and (d) The time-averaged density and instantaneous relative phases of individual condensates. Relative phases are plotted for times depicted with red dots in (a) and (c), respectively. The clusters of synchronized condensates are indicated by dashed circles of the same color. The numerical parameters in (a) and (b) are the same as in Fig. 2 with $P_{0}=32$, and the parameters in (c) and (d) are $g=0.1, b_{0}=0.05, b_{1}=200, \eta=0.01, w=0.8$, $P(\mathbf{r})=\sum_{i=1}^{N} P_{0} \exp \left[-w\left(\mathbf{r}-\mathbf{r}_{i}\right)^{2}\right], P_{0}=100 ; t_{0}$ is about $200 \mathrm{ps}$ in both cases.

Figs. 3(b) and 3(d) with dashed circles of the same color. The cluster state in Figs. 3(a) and 3(b) is a long-lived transient state that after a few nanoseconds evolves into chaotic oscillations. However, both reported states are stable against the addition of the random noise and against intrinsic roughness of the sample modeled by adding the noise potential to the right-hand side of Eq. (1) and therefore may be detected experimentally. The dimensionless parameters listed in the caption of Fig. 3 correspond to a polariton lifetime of $10 \mathrm{ps}$, exciton reservoir lifetime of $400 \mathrm{ps}, U_{0}=0.2 \mu \mathrm{eV} \mu \mathrm{m}^{2}$ and $g_{R}=2 \mu \mathrm{eV} \mu \mathrm{m}^{2}$ (the photonic component of polaritons dominates over their excitonic component), polariton mass $m=0.4 \mathrm{meV} \mathrm{ps}{ }^{2} / \mu \mathrm{m}^{2}$, and $R_{R}=20 g_{R}$. All found regimes are robust and can be demonstrated for reasonably different sets of parameters.

The established links between polaritonic networks and other coupled oscillators systems suggest that these two examples are infinitely far from describing all possible dynamical regimes and hint that other intriguing states can be obtained. A particular interest is in finding chimera states. A chimera state is a spatiotemporal pattern in a network of identical coupled oscillators in which some of the oscillators synchronize (become frequency locked) while others remain incoherent (desynchronized) [81,82]. The simplest model that supports such states was reported as a pair of SakaguchiKuramoto oscillator populations in which each oscillator has a different coupling to others in the same group than to those in another group [83]. The type of the pattern depends strongly on the parameter $g$ and therefore the phase lag $\alpha$. The spiral pattern of chimeras appears when $\alpha$ is close to zero, whereas the spot chimeras only appear when $\alpha$ is close to $\pi / 2$ [84]. The "turbulent chimeras" were observed in some special cases of nonlocally coupled Stuart-Landau oscillators in which regions of local synchronization appeared and vanished randomly over time [85]. The Stuart-Landau system equation (5) has also been shown to produce a wealth of various stationary and dynamical behaviors, e.g., amplitude death, Hopf oscillations, large oscillations, quasiperiodicity, and chaos [86]. Polaritonic networks are a promising platform to study the wealth of these phenomena.

To summarize, we proposed polaritonic networks as a paradigm for the dynamics of disparate systems of coupled oscillators. The dynamics of coupled oscillators often appear in the context of lasers and other driven-dissipative systems. It is therefore not surprising that systems of coupled oscillators are closely related to polaritonic systems. However, we showed that depending on the system parameters and experimental controls used such networks not only can reproduce the behavior of various known coupled oscillators and allow one to address intermediate regimes between different types of systems but also can dramatically increase the physics of the system by combining different types of oscillators together in one interacting platform. This opens intriguing possibilities for entering novel hybrid regimes that have not been implemented before. The polaritonic network is a flexible and robust platform for achieving this.

\section{ACKNOWLEDGMENTS}

N.G.B. acknowledges financial support from the NGP MIT-Skoltech. K.P.K. acknowledges the financial support from Cambridge Trust and EPSRC.

The authors declare that they have no competing interests.
[1] Y. Kuramoto, Chemical Oscillations, Waves, and Turbulence (Dover, New York, 2012).

[2] Y. Kuramoto, Self-entrainment of a population of coupled non-linear oscillators, International Symposium on Mathematical Problems in Theoretical Physics (Springer, Berlin, 1975), pp. 420-422.

[3] P. Hohenberg and B. Halperin, Rev. Mod. Phys. 49, 435 (1977).

[4] L. P. Kadanoff, Statistical Physics: Statics, Dynamics and Renormalization (World Scientific, Singapore, 2000).

[5] N. G. Berloff and J. Keeling, Universality in modelling nonequilibrium pattern formation in polariton condensates, in Physics of Quantum Fluids (Springer, Berlin, 2013), pp. 19-38.
[6] I. M. Georgescu, S. Ashhab, and F. Nori, Rev. Mod. Phys. 86, 153 (2014).

[7] T. Schwartz, G. Bartal, S. Fishman, and M. Segev, Nature (London) 446, 52 (2007).

[8] D. Bajoni, P. Senellart, E. Wertz, I. Sagnes, A. Miard, A. Lemaître, and J. Bloch, Phys. Rev. Lett. 100, 047401 (2008).

[9] J. Kasprzak, M. Richard, S. Kundermann, A. Baas, P. Jeambrun, J. M. J. Keeling, F. M. Marchetti, M. H. Szymaéska, R. Andre, J. L. Staehli, and V. Savona, Nature (London) 443, 409 (2006).

[10] C. Schneider, A. Rahimi-Iman, N. Y. Kim, J. Fischer, I. G. Savenko, M. Amthor, M. Lermer, A. Wolf, L. Worschech, V. D. 
Kulakovskii, and I. A. Shelykh, Nature (London) 497, 348 (2013).

[11] M. Saba, Nature (London) 414, 731 (2001).

[12] K. J. Vahala, Nature (London) 424, 839 (2003).

[13] H. Deng, G. Weihs, D. Snoke, J. Bloch, and Y. Yamamoto, Proc. Natl. Acad. Sci. USA 100, 15318 (2003).

[14] R. Balili, V. Hartwell, D. Snoke, L. Pfeiffer, and K. West, Science 316, 1007 (2007).

[15] G. Christmann, R. Butte, E. Feltin, J. F. Carlin, and N. Grandjean, Appl. Phys. Lett. 93, 051102 (2008).

[16] S. Kena-Cohen and S. R. Forrest, Nat. Photon. 4, 371 (2010).

[17] T. C. Lu, J. R. Chen, S. C. Lin, S. W. Huang, S. C. Wang, and Y. Yamamoto, Nano Lett. 11, 2791 (2011).

[18] C. Schneider, K. Winkler, M. D. Fraser, M. Kamp, Y. Yamamoto, E. A. Ostrovskaya, and S. Hofling, Rep. Prog. Phys. 80, 016503 (2017).

[19] A. Amo and J. Bloch, C. R. Phys. 17, 934 (2016).

[20] E. A. Cerda-Mendez, D. N. Krizhanovskii, K. Biermann, R. Hey, M. S. Skolnick, and P. V. Santos, Phys. Rev. B 86, 100301(R) (2012).

[21] N. Y. Kim, K. Kusudo, C. Wu, N. Masumoto, A. Loffler, S. Hofling, N. Kumada, L. Worschech, A. Forchel, and Y. Yamamoto, Nat. Phys. 7, 681 (2011).

[22] S. Dufferwiel, F. Fras, A. Trichet, P. M. Walker, F. Li, L. Giriunas, M. N. Makhonin, L. R. Wilson, J. M. Smith, E. Clarke, and M. S. Skolnick, Appl. Phys. Lett. 104, 192107 (2014).

[23] K. Winkler, J. Fischer, A. Schade, M. Amthor, R. Dall, J. Geßler, M. Emmerling, E. A. Ostrovskaya, M. Kamp, C. Schneider, and S. Hofling, New J. Phys. 17, 023001 (2015).

[24] T. Boulier, M. Bamba, A. Amo, C. Adrados, A. Lemaitre, E. Galopin, I. Sagnes, J. Bloch, C. Ciuti, E. Giacobino, and A. Bramati, Nat. Commun. 5, 3260 (2014).

[25] N. Y. Kim, K. Kusudo, A. Loffler, S. Hofling, A. Forchel, and Y. Yamamoto, New J. Phys. 15, 035032 (2013).

[26] K. Kusudo, N. Y. Kim, A. Loffler, S. Hofling, A. Forchel, and Y. Yamamoto, Phys. Rev. B 87, 214503 (2013).

[27] T. Jacqmin, I. Carusotto, I. Sagnes, M. Abbarchi, D. D. Solnyshkov, G. Malpuech, E. Galopin, A. Lemaitre, J. Bloch, and A. Amo, Phys. Rev. Lett. 112, 116402 (2014).

[28] N. Masumoto, N. Y. Kim, T. Byrnes, K. Kusudo, A. Loffler, S. Hofling, A. Forchel, and Y. Yamamoto, New J. Phys. 14, 065002 (2012).

[29] D. Tanese, E. Gurevich, F. Baboux, T. Jacqmin, A. Lemaître, E. Galopin, I. Sagnes, A. Amo, J. Bloch, and E. Akkermans, Phys. Rev. Lett. 112, 146404 (2014).

[30] N. G. Berloff, M. Silva, K. Kalinin, A. Askitopoulos, J. D. Topfer, P. Cilibrizzi, W. Langbein, and P. G. Lagoudakis, Nat. Mater. 16, 1120 (2017).

[31] E. Wertz, L. Ferrier, D. D. Solnyshkov, R. Johne, D. Sanvitto, A. Lemaitre, I. Sagnes, R. Grousson, A. V. Kavokin, P. Senellart, and G. Malpuech, Nat. Phys. 6, 860 (2010).

[32] F. Manni, K. G. Lagoudakis, T. C. H. Liew, R. André, and B. Deveaud-Plédran, Phys. Rev. Lett. 107, 106401 (2011).

[33] G. Tosi, G. Christmann, N. G. Berloff, P. Tsotsis, T. Gao, Z. Hatzopoulos, P. G. Savvidis, and J. J. Baumberg, Nat. Phys. 8, 190 (2012).

[34] G. Tosi, G. Christmann, N. G. Berloff, P. Tsotsis, T. Gao, Z. Hatzopoulos, P. G. Savvidis, and J. J. Baumberg, Nat. Commun. 3, 1243 (2012).
[35] J. Keeling and N. G. Berloff, Phys. Rev. Lett. 100, 250401 (2008).

[36] M. Wouters and I. Carusotto, Phys. Rev. Lett. 99, 140402 (2007).

[37] I. Carusotto and C. Ciuti, Rev. Mod. Phys. 85, 299 (2013).

[38] J. Keeling and N. G. Berloff, Contemp. Phys. 52, 131 (2011).

[39] P. Cristofolini, A. Dreismann, G. Christmann, G. Franchetti, N. G. Berloff, P. Tsotsis, Z. Hatzopoulos, P. G. Savvidis, and J. J. Baumberg, Phys. Rev. Lett. 110, 186403 (2013).

[40] H. Ohadi, A. Dreismann, Y. G. Rubo, F. Pinsker, Y. del Valle-Inclan Redondo, S. I. Tsintzos, Z. Hatzopoulos, P. G. Savvidis, and J. J. Baumberg, Phys. Rev. X 5, 031002 (2015).

[41] K. G. Lagoudakis, M. Wouters, M. Richard, A. Baas, I. Carusotto, R. Andre, L. S. Dang, and B. Deveaud-Pledran, Nat. Phys. 4, 706 (2008).

[42] K. G. Lagoudakis, B. Pietka, M. Wouters, R. Andre, and B. Deveaud-Pledran, Phys. Rev. Lett. 105, 120403 (2010).

[43] T. Gao, E. Estrecho, K. Y. Bliokh, T. C. H. Liew, M. D. Fraser, S. Brodbeck, M. Kamp, C. Schneider, S. Hofling, Y. Yamamoto, and F. Nori, Nature (London) 526, 554 (2015).

[44] M. D. Fraser, G. Roumpos, and Y. Yamamoto, New J. Phys. 11, 113048 (2009).

[45] D. Sanvitto, F. M. Marchetti, M. H. Szymanska, G. Tosi, M. Baudisch, F. P. Laussy, D. N. Krizhanovskii, M. S. Skolnick, L. Marrucci, A. Lemaitre, and J. Bloch, Nat. Phys. 6, 527 (2010).

[46] M. Wouters, New J. Phys. 14, 075020 (2012).

[47] B. Nelsen, G. Liu, M. Steger, D. W. Snoke, R. Balili, K. West, and L. Pfeiffer, Phys. Rev. X 3, 041015 (2013).

[48] K. P. Kalinin and N. G. Berloff, New J. Phys. 20, 113023 (2018).

[49] J. Ch. Eilbeck, P. S. Lomdahl, and A. C. Scott, Phys. D (Amsterdam, Neth.) 16, 318 (1985).

[50] G. L. Alfimov, P. G. Kevrekidis, V. V. Konotop, and M. Salerno, Phys. Rev. E 66, 046608 (2002).

[51] A. Trombettoni and A. Smerzi, Phys. Rev. Lett. 86, 2353 (2001).

[52] N. Nakagawa and Y. Kuramoto, Prog. Theor. Phys. 89, 313 (1993); Phys. D (Amsterdam, Neth.) 75, 74 (1994).

[53] H. Sakaguchi and Y. Kuramoto, Prog. Theor. Phys. 76, 576 (1986).

[54] L. Q. English, Z. Zeng, and D. Mertens, Phys. Rev. E 92, 052912 (2015).

[55] G. Filatrella, A. H. Nielsen, and N. F. Pedersen, Eur. Phys. J. B 61, 485 (2008).

[56] K. Vasudevan, M. Cavers, and A. Ware, Nonlinear Processes Geophys. 22, 499 (2015).

[57] H. K. Khalil, Nonlinear Systems, 3rd ed. (Prentice Hall, Upper Saddle River, 2002).

[58] P. Monzón and F. Paganini, in Proceedings of the 44th IEEE Conference on Decision and Control (IEEE, Picasway, 2005), pp. 3923-3928.

[59] P. G. Lagoudakis and N. G. Berloff, New J. Phys. 19, 125008 (2017).

[60] K. P. Kalinin and N. G. Berloff, Phys. Rev. Lett. 121, 235302 (2018).

[61] S. Utsunomiya, K. Takata, and Y. Yamamoto, Opt. Express 19, 18091 (2011).

[62] A. Marandi, Z. Wang, K. Takata, R. L. Byer, and Y. Yamamoto, Nat. Photon. 8, 937 (2014). 
[63] T. Inagaki, K. Inaba, R. Hamerly, K. Inoue, Y. Yamamoto, and H. Takesue, Nat. Photon. 10, 415 (2016).

[64] P. L. McMahon, A. Marandi, Y. Haribara, R. Hamerly, C. Langrock, S. Tamate, T. Inagaki, H. Takesue, S. Utsunomiya, K. Aihara, and R. L. Byer, Science 354, 614 (2016).

[65] Y. Takeda, S. Tamate, Y. Yamamoto, H. Takesue, T. Inagaki, and S. Utsunomiya, Quantum Sci. Technol. 3, 014004 (2017).

[66] M. Nixon, E. Ronen, A. A. Friesem, and N. Davidson, Phys. Rev. Lett. 110, 184102 (2013).

[67] D. Dung, C. Kurtscheid, T. Damm, J. Schmitt, F. Vewinger, M. Weitz, and J. Klaers, Nat. Photon. 11, 565 (2017).

[68] E. Estrecho, T. Gao, N. Bobrovska, D. Comber-Todd, M. D. Fraser, M. Steger, K. West, L. N. Pfeiffer, J. Levinsen, M. M. Parish, T. C. H. Liew, M. Matuszewski, D. W. Snoke, A. G. Truscott, and E. A. Ostrovskaya, Phys. Rev. B 100, 035306 (2019).

[69] B. Eckhardt, E. Ott, S. H. Strogatz, D. M. Abrams, and A. McRobie, Phys. Rev. E 75, 021110 (2007).

[70] I. Kiss, Y. Zhai, and J. Hudson, Science 296, 1676 (2002).

[71] R. Lang and K. Kobayashi, IEEE J. Quantum Electron. 16, 347 (1980).

[72] J. A. Acebrón, L. L. Bonilla, C. J. P. Vicente, F. Ritort, and R. Spigler, Rev. Mod. Phys. 77, 137 (2005).

[73] K. P. Kalinin, P. G. Lagoudakis, and N. G. Berloff, Phys. Rev. B 97, 161101(R) (2018).

[74] K. P. Kalinin, P. G. Lagoudakis, and N. G. Berloff, Phys. Rev. B 97, 094512 (2018).
[75] A. Fert, C. Vincent, and S. Joao, Nat. Nanotechnol. 8, 152 (2013).

[76] K. G. Lagoudakis, F. Manni, B. Pietka, M. Wouters, T. C. H. Liew, V. Savona, A. V. Kavokin, R. Andre, and B. DeveaudPledran, Phys. Rev. Lett. 106, 115301 (2011).

[77] D. Ballarini, I. Chestnov, D. Caputo, M. De Giorgi, L. Dominici, K. West, L. N. Pfeiffer, G. Gigli, A. Kavokin, and D. Sanvitto, Phys. Rev. Lett. 123, 047401 (2019).

[78] D. Ballarini, M. De Giorgi, E. Cancellieri, R. Houdre, E. Giacobino, R. Cingolani, A. Bramati, G. Gigli, and D. Sanvitto, Nat. Commun. 4, 1778 (2013).

[79] K. Kaneko, Phys. D (Amsterdam, Neth.) 75, 55 (1994).

[80] A. Hu, J. Cao, M. Hu, and L. Guo, Phys. A (Amsterdam, Neth.) 395, 537 (2014).

[81] O. E. Omel'chenko, M. Wolfrum, S. Yanchuk, Y. L. Maistrenko, and O. Sudakov, Phys. Rev. E 85, 036210 (2012).

[82] Y. Kuramoto and D. Battogtokh, Nonlinear Phenom. Complex Syst. (Minsk, Belarus) 5, 380 (2002).

[83] D. M. Abrams, R. Mirollo, S. H. Strogatz, and D. A. Wiley, Phys. Rev. Lett. 101, 084103 (2008).

[84] M. J. Panaggio and D. M. Abrams, Nonlinearity 28, R67 (2015).

[85] G. Bordyugov, A. Pikovsky, and M. Rosenblum, Phys. Rev. E 82, 035205(R) (2010).

[86] P. C. Matthews, R. E. Mirollo, and S. H. Strogatz, Phys. D (Amsterdam, Neth.) 52, 293 (1991). 